\title{
Eine neue Fachgesellschaft?
}

\section{Carlos Beat Quinto}

Dr. med., Mitglied des FMH-Zentralvorstandes, Departementsverantwortlicher Public Health und Gesundheitsberufe

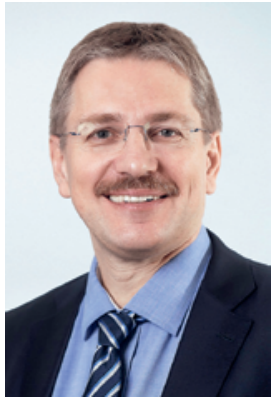

Die Schweizerische Facharztgesellschaft für Prävention und Gesundheitswesen gibt sich einen neuen Namen: Schweizerische Fachgesellschaft für Prävention und Public Health. Was neben der Namensänderung alles zum Tätigkeitsfeld dieser Fachärztinnen und -ärzte gehört, erfahren Sie in dieser Ausgabe. Gesundheit und Krankheit sollten immer aus der Individual- und aus der Bevölkerungsperspektive gedacht werden. Der Perspektivenwechsel ist nicht konfliktfrei und erfordert sowohl Kenntnisse in klinischer, auf das Individuum ausgerichteter Medizin als auch eine Public-HealthAusbildung. Ebenso sind Verhaltens- und Verhältnisprävention immer in Kombination durchzuführen - alles andere ist weder stimmig noch effizient. Es gibt eine grosse gemeinsame Schnittmenge in den Bereichen Primärversorgung und Public Health. Welche Probleme gilt es zu lösen? Gerade im Bereich Verhältnisprävention hinsichtlich Alkohol und Tabak gibt es in der Schweiz viel Luft nach oben. Es liessen sich Gesundheitskosten und Zahlungen an die Krankenkassen in Milliardenhöhe einsparen. Über 50 europäische Länder sind diesbezüglich weiter. Wir wollen es uns politisch von Seiten des Parlamentes vorderhand noch leisten, notwendigerweise Milliarden von Franken jährlich in der Gesundheitsversorgung auszugeben, die sich durch einfache verhältnispräventive Massnahmen einsparen liessen, ohne dass auch nur irgendeinem Patienten irgendwelche

\section{Primärversorgung und Public Health haben viele Gemeinsamkeiten: Es geht darum, eine Dehumanisierung der Medizin zu vermeiden.}

Leistungen vorenthalten werden müssten. Es braucht kein Globalbudget und keine Rationierung, wohl aber Zielvorgaben im Bereich Verhältnisprävention, um nicht als unglaubwürdig und korrupt wahrgenommen zu werden. Beim Kostenproblem ist festzuhalten, dass wir in den letzten 10 Jahren eine Explosion der Krankenkassenprämien, aber keine der Gesundheitskosten haben, wenn als Referenz die Zahlen des Bundesamtes für Statistik (MOKKE) genommen werden. Der Prämienanstieg, der sich ideal als politisches Druckmittel einsetzen lässt, liegt wesentlich über dem effektiven Anstieg der Gesundheitskosten. Die Facharztgesellschaft für Prävention und Public Health kümmert sich auch um die Schnittstelle zwischen Sozialwissenschaften und Medizin, was Thema der diesjährigen Schweizer Public-Health-Konferenz war. Advocacy tut not. Das Erlernen dieser Rolle und die dafür notwendigen Fähigkeiten sind integraler Bestandteil von PROFILES, dem neuen Lernzielkatalog der medizinischen Fakultäten der Schweiz. Falschen ökonomischen Annahmen, die unbedarft in Anlehnung an Produktionsprozesse aus der Betriebsökonomie übernommen werden,

Die essentielle ärztliche Leistung, den kranken Menschen zu verstehen, lässt sich nicht in Zahlen messen.

ist entgegenzutreten. Sie führen zur Schaffung von Fehlanreizen, gefährden zentrale ethische Werte der Medizin und schädigen die intrinsische Motivation der Angehörigen aller Medizinal- und Gesundheitsberufe. Dies kann nur im Dialog geschehen. Strategien sind nicht für die Ärzteschaft, sondern im Sinne einer adäquaten Partizipation mit der Ärzteschaft zu entwickeln. Andernfalls wird sich die Kluft, die sich infolge der demographischen Entwicklung und eines Fachkräftemangels öffnet, weiter zunehmen. Der aus einer Kultur des Misstrauens entstehende administrative Overkill, der niemals leisten kann, was er verspricht, nämlich Sicherheit, führt bereits heute zu schwerwiegenden Nebenwirkungen. Es geht gemäss Giovanni Maio darum, eine Dehumanisierung der Medizin zu vermeiden. Hierfür lohnt sich ein gemeinsamer, interprofessioneller Ansatz. Patientinnen und Patienten sind kein Stückgut. Die essentielle ärztliche Leistung, den kranken Menschen zu verstehen, lässt sich gemäss Giovanni Maio nicht einfach messen. In diesem Sinne ist das Departement Public Health und Gesundheitsberufe der FMH an einer Fortsetzung der bisherigen guten Zusammenarbeit mit der Schweizerischen Fachgesellschaft für Prävention und Public Health interessiert. 